\title{
Role of Immunomodulation of BCG Therapy on AML Remission
}

This article was published in the following Dove Press journal:

International Medical Case Reports Journal

\author{
Alice Kennedy' \\ Kamal Kant Sahu (iD ${ }^{2}$ \\ Jan Cerny ${ }^{3}$ \\ 'Department of Internal Medicine, \\ University of Massachusetts Medical \\ School, Worcester, MA, 01655, USA; \\ ${ }^{2}$ Department of Internal Medicine, Saint \\ Vincent Hospital, Worcester, MA, 01608, \\ USA; ${ }^{3}$ Division of Hematology and \\ Oncology, Department of Internal \\ Medicine, UMass Memorial Health Care, \\ University of Massachusetts Medical \\ School, Worcester, MA, 01655, USA
}

Correspondence: Jan Cerny Division of Hematology and Oncology, Department of Internal Medicine, UMass Memorial Health Care, University of Massachusetts Medical School, Worcester, MA, 01655, USA

Email Jan.cerny@umassmemorial.org

\begin{abstract}
Bacillus Calmette-Guérin (BCG) has been studied in various cancers for its immune modulation. Although the mechanism is yet to be completely understood, we do have positive experiences in many oncological cases. Hereby, we present a case of an 82-year-old male with acute myeloid leukemia (AML) post allogeneic Stem Cell Transplants (AlloSCT) as a salvage therapy, now in remission, who presented with hematuria. Workup confirmed non-muscle invasive Bladder Cancer (NMIBC), for which he was treated with nine sessions of intravesical BCG therapy. During BCG treatment, the patient developed gradually increasing lymphocyte counts. Flowcytometry of a peripheral blood sample showed polyclonal cell lymphocytosis with CD8+ T-cell expansion. Although further work up his lymphocytosis to be polyclonal, it has persisted at follow-up for the last 4 years. Also, we did not find any evidence of leukemia recurrence at follow-up prompting us to associate the BCG use for this patient and it is role as immunomodulation to keep AML disease in remission.
\end{abstract}

Keywords: cancer, bladder, leukemia, vaccine, immunity

\section{Introduction}

The Mycobacterium bovis strain, Bacillus Calmette-Guérin (BCG) is an attenuated, live vaccine developed to prevent tuberculosis. There is evidence to suggest its role as a cancer-fighting immunotherapy, and previously BCG has been used in the treatment of various malignancies such as leukemia and melanoma. ${ }^{1,2}$ Nowadays, intravesical administration of BCG for non-muscle invasive Bladder Cancer (NMIBC) is standard of care as it reduces the risk of recurrence and progression. ${ }^{3,4}$

A robust inflammatory infiltrate and T-cell expansion within bladder-draining lymph nodes is characteristic of responders to BCG with bladder cancer. ${ }^{5,6}$ Effector CD4+ T cells and CD8+ T-cells are known to be essential for tumor reduction. However, whether crossantigenicity or another mechanism accounts for this effect remains under active investigation. In addition, past studies on systemic BCG therapy have demonstrated its ability towards immune reconstitution against leukemia-associated antigen (LAA) or leukemiaspecific antigens (LSA). In the past, scientists also attempted to develop a vaccine against Acute Myeloid Leukemia (AML) via epigenetic reprogramming. ${ }^{7}$ However, so far, vaccine against AML has only shown an increase in remission duration during the maintenance phase following induction and consolidation. ${ }^{7}$

Neither BCG nor other immunotherapies are currently recommended for treatment of AML during any phase. However, AML disease severity has been inversely correlated with the effectiveness of the immune response. ${ }^{8}$ Here, we describe a survivor of NMIBC and AML with persistent lymphocytosis 4 years after BCG therapy. He has no evidence of leukemia relapse or bladder cancer progression or recurrence. 


\section{Case Presentation}

A 78-year-old gentleman presented to our hospital 16 years ago for fever and shortness of breath of 2-week duration. Laboratory workup suggested pancytopenia with circulating blast cells. A bone marrow aspirate with biopsy was requested which was consistent with AML with multilineage dysplasia. Subsequently, the patient was treated with daunorubicin/Ara-C "7+3" induction chemotherapy. Repeat bone marrow biopsy on day 28 confirmed a complete remission (CR). This was followed by one cycle of consolidation therapy with high dose Ara-C and autologous Hemopoietic Stem Cell Transplant (HSCT) without complications.

A year later, the patient relapsed, as evidenced by pancytopenia, and had $35 \%$ blasts in the bone marrow examination. This time, he was treated with two cycles of the FLAG (fludarabine, cytarabine and filgrastim) regimen. This resulted in a 2nd CR that lasted until January 2009 when biopsy revealed tetraploid karyotype with multiple chromosomal abnormalities. High dose Mitoxantrone/Cytarabine salvage therapy achieved a 3rd CR followed by a repeat autologous HSCT. His bone marrow biopsy showed $1 \%$ blasts and cytogenetics revealed minimal residual disease 3 months following HSCT. The patient was followed at a regular interval for disease monitoring.

Five years after 2nd HSCT, the patient's peripheral blood cell counts started to fall again, and $56 \%$ blasts were noted in the bone marrow. Salvage therapy with decitabine was initiated for his refractory disease and the patient received four cycles of injection decitabine without severe adverse effects. He then underwent an allogeneic stem cell transplantation in CR4. His posttransplant course was complicated by reactivated Cytomegalovirus (CMV) antigenemia treated with ganciclovir, and BK virus induced urinary tract infections treated symptomatically with pyridium.

For nearly the following 4 years, the patient continued to remain in remission from AML but developed hematuria as confirmed by urinary cytology. Pathology from his subsequent Transurethral Resection of Bladder Tumor (TURBT) showed G1 papillary carcinoma limited to the bladder lining, stage pTa. He was followed with every 3 months surveillance cystoscopy (Figure 1).

He remained asymptomatic for the next 1 year when urinary symptoms returned, and five bladder tumors were identified. He underwent TURBT with bilateral retrograde pyelograms without any complications that revealed that the tumors were of high-grade squamous differentiation without muscle invasion, stage pT1. As the patient was now immunocompetent with recurrent pT1 superficial bladder cancer, an induction regimen of six weekly intravesical administrations of BCG was planned. Although his fourth treatment was delayed due to the development of a non-mycobacterial pneumonia, the patient successfully completed the treatments without severe adverse effects.

Follow-up cystoscopy after completion of the BCG therapy showed no residual disease. Laboratory results at this time included WBC count: $11.7 \times 10^{3} / \mu \mathrm{L}\left(4.3-10.8 \times 10^{3} / \mu \mathrm{L}\right), \mathrm{RBC}$ count: $3.63 \times 10^{6} / \mu \mathrm{L}\left(4.2-5.8 \times 10^{6} / \mu \mathrm{L}\right)$, platelet count: $119 \times 10^{3} /$ $\mu \mathrm{L} \quad\left(140-440 \times 10^{3} / \mu \mathrm{L}\right)$, and absolute lymphocyte count: $5.6 \times 10^{3} / \mu \mathrm{L}\left(0.9-3.4 \times 10^{3} / \mu \mathrm{L}\right)$. In the context of lymphocytosis, a peripheral blood flow cytometry was performed, which ruled out co-existent chronic lymphoproliferative disorders. Urinary symptoms and fatigue were stable allowing for three additional BCG treatments as consolidation therapy.

Lymphocytosis peaked at $12 \times 10^{3}$ cells $/ \mu \mathrm{L}$, approximately 6 months following his last BCG treatment (Figure 2). A repeat flow cytometry confirmed a T-cell predominance with $5432 \mathrm{CD} 3+$ cells $/ \mu \mathrm{L}(710-2300$ cells $/ \mu \mathrm{L})$ and a CD4:CD8 ratio of $0.22(0.8-5.0)$. So far, the patient remains clinically asymptomatic without lymphadenopathy and in AML remission. The patient is being followed closely by hematology and urology with no further evidence of disease recurrence of AML or bladder cancer. Most recent flow cytometry done in September 2020 was consistent with chronic CD3+ and CD8+ expansion; $5712 \mathrm{CD} 3+$ cells $/ \mu \mathrm{L}(710-2300$ cells/ $\mu \mathrm{L})$ with a CD4:CD8 ratio of $0.2(0.8-5.0)$ (Table 1).

\section{Discussion}

Our case highlights the immunomodulating character of BCG in the context of AML. Recently, immunotherapy has been a widely accepted modality for a variety of cancers. ${ }^{9}$ Various microorganisms have been investigated as immunomodulatory agents, including BCG. The best evidence for its efficacy is its activity against NMIBC for which it is a standard of care. ${ }^{10}$

In our case, for high-grade NMIBC, our patient received 6 weeks of induction and 3 weeks of maintenance intravesical BCG installations. Even with this considered optimal therapy schedule, however, $30-35 \%$ of superficial bladder do not respond to therapy or recur while on treatment. Optimizing treatment regimens to enhance response is an important area of contemporary research. ${ }^{11}$

Recent studies suggest that exposure to BCG induces epigenetic reprogramming in innate immune cells such as monocytes. ${ }^{12,13}$ When subjected to a subsequent pathogen 


\section{DISEASE TIMELINE OF PATIENT FROM DIAGNOSIS OF AML TILL LAST FOLLOW UP}

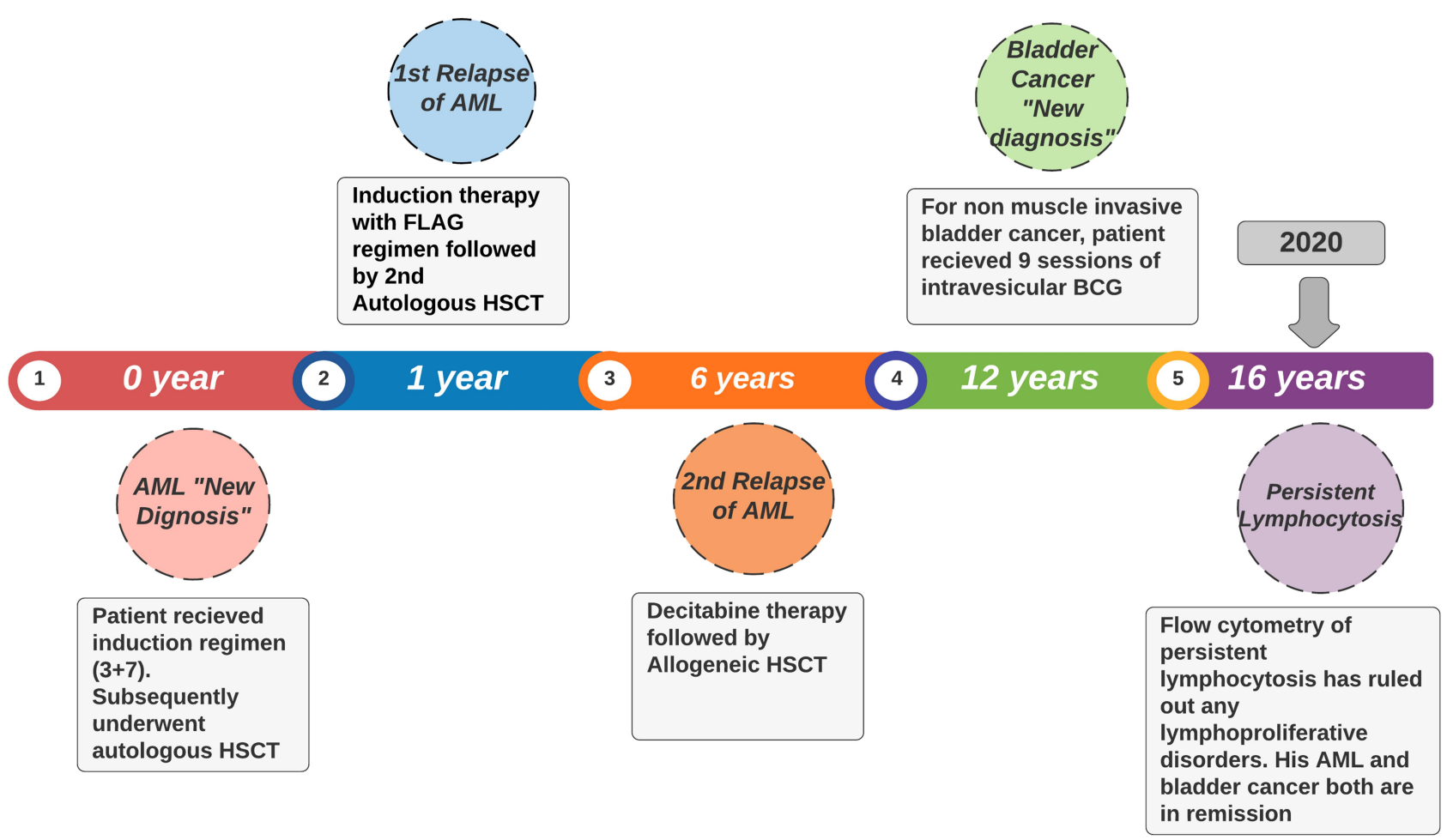

Figure I Disease timeline of the patient course of illness from the time of diagnosis of AML to last follow-up.

or cancerous process, these cells have altered metabolism and augmented cytokine release.

The process of immunomodulation caused by intravesical BCG begins when mycobacteria are internalized by urothelial cells by fibronectin and integrin receptors. ${ }^{14}$ Innate immune cells present BCG antigens, release cytokines, and mobilize a granulomatous infiltrate. Granulocytes, CD4+ and CD8+ $\mathrm{T}$ cells, natural killer (NK) cells and macrophages infiltrate the bladder, releasing a deluge of primarily TH-1 cytokines. ${ }^{6}$ Numerous downstream pathways are activated to achieve tumor cell killing.

In essence, $\mathrm{BCG}$ alters the bladder tissue microenvironment to reverse the immunosuppressive phenotype seen in bladder cancer. This allows innate immune cells to mount a vigorous secondary response against unrelated cancer cells. ${ }^{15}$ Stimulation of cytotoxic $\mathrm{T}$ lymphocytes and NK cells constitute as major contributors to promote anti-tumor effect following BCG therapy in case of bladder cancer. The use of BCG therapy is considered safe as BCG is live attenuated which considerably decreases its infection capability. But, there is a theoretical risk of increased morbidity and sepsis, especially in immunocompromised patients just like any naturally acquired mycobacteria infection. ${ }^{16}$ However, a previous report does describe the safe utilization of intravesical BCG in several post-HSCT patients with refractory NMIBC. ${ }^{17}$

The other facts to note in our case are the persistence of lymphocytosis (consisting mostly of $\mathrm{T}$ cells that were CD3 $+\mathrm{CD} 8+$ cell subtype) post BCG therapy and continued remission of AML disease. Prior to his NMIBC diagnosis, our patient was struggling with multiple relapses of his AML disease and required three HSCTs since the time of the first diagnosis in 2004. Blasts of AML are poorly immunogenic and have immune-evasive properties including suppression of the costimulatory ligands and MHC proteins required for immune activation. ${ }^{8}$ This is also one reason that patients who have delayed immune reconstitution following HSCT have a high risk of AML relapse. ${ }^{18}$ While there is ample evidence of immune failure in patients with AML, no immunotherapies are first-line treatment.

The success of HSCT depends on the Graft-vs-Leukemia effect when donor lymphocytes attack leukemic cells. This concept is utilized in some patients with Donor Lymphocyte Infusions (DLI). ${ }^{19,20}$ Similar functional expansion of CD8+ 


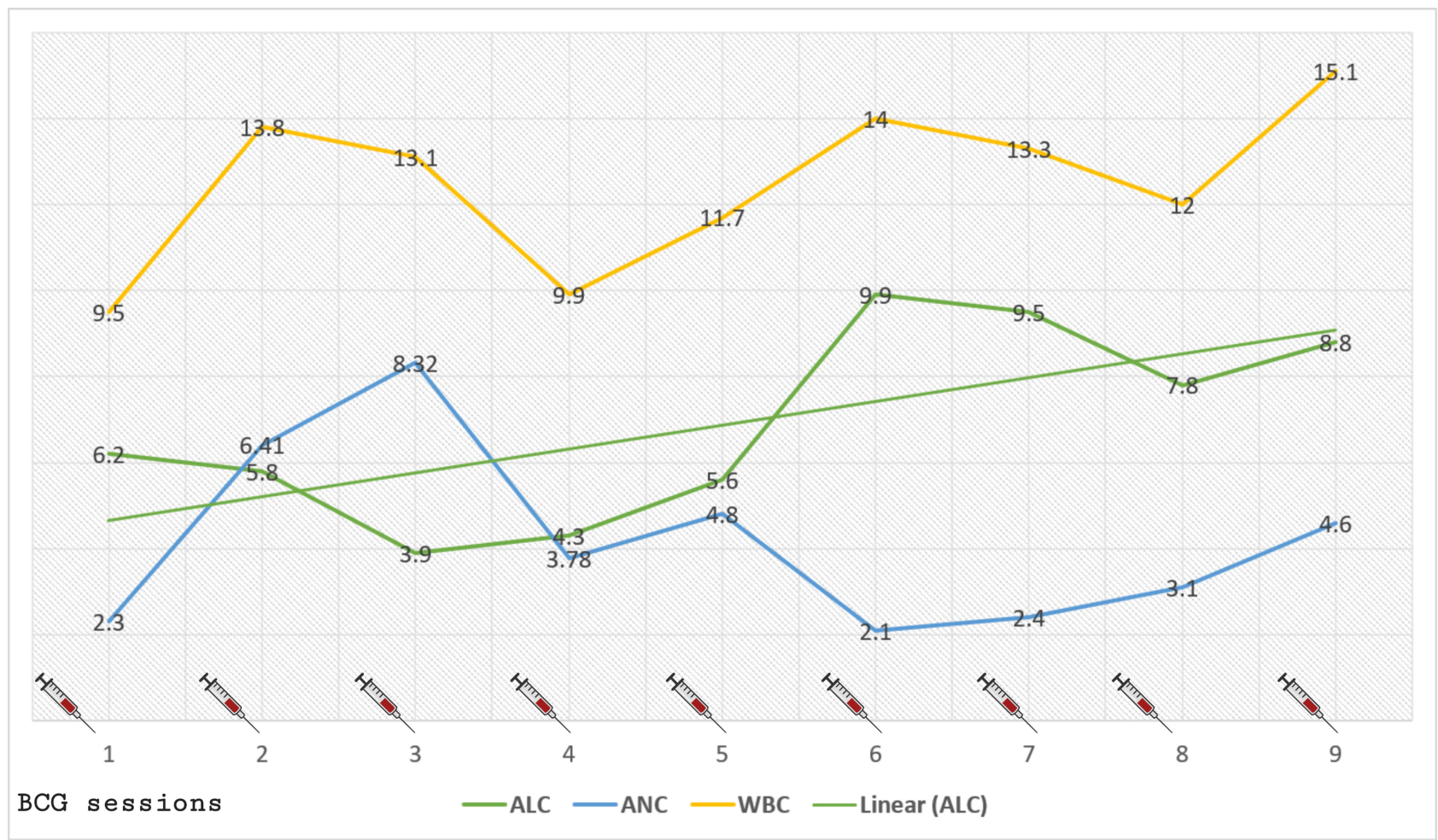

Figure 2 Trend of complete blood count parameters, and their association with BCG vaccinations. $\left(\times 10^{3}\right.$ cells $\left./ \mu \mathrm{L}\right)$ Abbreviations: WBC, whole blood count; ALC, absolute lymphocyte count; ANC, absolute neutrophil count.

T-cells with anti-leukemic effect has also been noted following BCG therapy. It has been proposed that BCG injection promotes anti-leukemic activity by activation of the immune system by increasing TNF-alpha, IFN-gamma, and IL-2. ${ }^{21}$ BCG acts as a Toll-like receptor agonist and stimulates TLR2 and TRL4. TLR activation in turn enhances NK cellmediated cytotoxicity, enhancing the MHC class I on leukemic cells, that ultimately leads to leukemic cell apoptosis. ${ }^{22}$ We hypothesize that the persistent lymphocytosis with T-cell predominance and Toll-like receptor agonism secondary to BCG therapy together with nonspecific effect of live vaccine

Table I Flow Cytometry Results at Various Intervals Showing CD3+ Predominant Lymphocytosis ( $\times$ cells $/ \mu \mathrm{L}$ )

\begin{tabular}{|l|l|l|}
\hline Date & CD3 & CD4:CD8 \\
\hline $3 / 5 / 2015$ & 3909 & 0.3 \\
$5 / 28 / 2015$ & 4514 & 0.38 \\
$7 / 28 / 2016$ & 7408 & 0.14 \\
$11 / 16 / 2016$ & 6760 & 0.19 \\
$5 / 24 / 2017$ & 5432 & 0.21 \\
$11 / 7 / 2018$ & 5328 & 0.18 \\
$4 / 29 / 2019$ & 6432 & 0.19 \\
$10 / 30 / 2019$ & 6160 & 0.18 \\
$9 / 9 / 2020$ & 5712 & 0.2 \\
\hline
\end{tabular}

or trained immunity contributed to continued anti-leukemic effect against AML in our patient.

\section{Conclusion}

Immuno-oncology is an evolving field in cancer medicine. Understanding of the complex mechanism of BCG therapy in modulating the immune system is the need of the hour. This case suggests the need for further basic and clinical research to develop new treatment strategies for patients suffering from various malignancies.

\section{Ethical Statement}

The article does not contain the participation of any human being and animal.

\section{Consent}

Informed consent was obtained from the patient to publish their case details and accompanying images.

\section{Author Contributions}

All authors contributed to data analysis, drafting or revising the article, have agreed on the journal to which the article will be submitted, gave final approval of the version to be published, and agree to be accountable for all aspects of the work. 


\section{Disclosure}

Dr Jan Cerny serves in the advisory board/consultancy for Jazz Pharmaceuticals and Amgen. He is aData and Safety Monitoring Board Member for AlloVir, and holds stocks from Actinium Pharmaceuticals, Bluebird Bio Inc., Dynavax Pharma, Atyr Pharmac, Gamida Cell, Miragen Therapeutics, Mustang Bio, Novavax, Ovid Therapeutics, Sorrento Therapeutics, TG Therapeutics, Vaxart Inc, and Veru Inc., outside the submitted work. The authors have no other conflicts of interest to declare.

\section{References}

1. Mathé G, Amiel JL, Schwarzenberg L, et al. Active immunotherapy for acute lymphoblastic leukaemia. Lancet. 1969;1(7597):697-699. doi:10.1016/s0140-6736(69)92648-8

2. Morton DL, Eilber FR, Holmes EC, et al. BCG immunotherapy of malignant melanoma: summary of a seven year experience. Ann Surg. 1974;180(4):635-643. doi:10.1097/00000658-197410000-00029

3. Han RF, Pan JG. Can intravesical bacillus Calmette-Guérin reduce recurrence in patients with superficial bladder cancer? A metaanalysis of randomized trials. Urology. 2006;67(6):1216-1223. doi:10.1016/j.urology.2005.12.014

4. Sylvester RJ, van der M, Lamm DL. Intravesical bacillus Calmette-Guerin reduces the risk of progression in patients with superficial bladder cancer: a meta-analysis of the published results of randomized clinical trials. J Urol. 2002;168(5). doi:10.1097/01. JU.0000034450.80198.1C

5. Prescott S, James K, Hargreave TB, Chisholm GD, Smyth JF. Intravesical Evans strain BCG therapy: quantitative immunohistochemical analysis of the immune response within the bladder wall. $J$ Urol. 1992;147(6):1636-1642. doi:10.1016/S0022-5347(17)37668-1

6. Alhunaidi O, Zlotta AR. The use of intravesical BCG in urothelial carcinoma of the bladder. Ecancermedicalscience. 2019;13. doi:10.3332/ecancer.2019.905.

7. Anguille S, Van Tendeloo VF, Berneman ZN. Leukemia-associated antigens and their relevance to the immunotherapy of acute myeloid leukemia. Leukemia. 2012;26(10):2186-2196. doi:10.1038/ leu. 2012.145

8. Teague RM, Kline J. Immune evasion in acute myeloid leukemia: current concepts and future directions. J Immunother Cancer. 2013;1 (1): 1. doi:10.1186/2051-1426-1-13

9. Lal A, Sahu KK, Jindal V, Mishra AK, Siddiqui AD. Role of immunotherapy in metastatic renal cell cancer: past, present and future. Ann Transl Med. 2019;7(S8):S349-S349. doi:10.21037/atm.2019.09.95
10. Akamat AMK, Org M, Kamat AM, et al. Expert consensus document: consensus statement on best practice management regarding the use of intravesical immunotherapy with BCG for bladder cancer. Nat Rev Urol. 2015;12:225-235. doi:10.1038/nrurol.2015.58

11. Morales A. BCG: a throwback from the stone age of vaccines opened the path for bladder cancer immunotherapy. Can J Urol. 2017;24 (3):8788-8793.

12. Buffen K, Oosting M, Quintin J, et al. Autophagy controls BCG-induced trained immunity and the response to intravesical BCG therapy for bladder cancer. PLoS Pathog. 2014;10(10): e1004485. doi:10.1371/journal.ppat.1004485

13. Kleinnijenhuis J, Quintin J, Preijers F, et al. Bacille Calmette-Guérin induces NOD2-dependent nonspecific protection from reinfection via epigenetic reprogramming of monocytes. Proc Natl Acad Sci U S A. 2012;109(43):17537-17542. doi:10.1073/pnas.1202870109

14. Zhao W, Schorey JS, Groger R, Allen PM, Brown EJ, Ratliff TL. Characterization of the fibronectin binding motif for a unique mycobacterial fibronectin attachment protein, FAP. J Biol Chem. 1999;274 (8):4521-4526. doi:10.1074/jbc.274.8.4521

15. Joseph M, Enting D. Immune responses in bladder cancer-role of immune cell populations, prognostic factors and therapeutic implications. Front Oncol. 2019;9:1270. doi:10.3389/ fonc. 2019.01270

16. Sahu KK, Mishra AK, Lal A, Abraham GM. Mycobacterium Avium Complex: A Rare Cause of Pancytopenia in HIV Infection. J Microsc Ultrastruct. 2019;8(1):27-30

17. Petropoulou AD, Lapusan S, Marie JP, Rio B. Intravesical Bacillus Calmette-Guérin for the treatment of superficial bladder cancer following hematopoietic stem cell transplantation. Leuk Lymphoma. 2008;49(8):1627-1629. doi:10.1080/10428190802136376

18. Ohnishi K, Yamanishi H, Naito K, et al. Reconstitution of peripheral blood lymphocyte subsets in the long-term disease-free survivors of patients with acute myeloblastic leukemia. Leukemia. 1998;12 (1):52-58. doi:10.1038/sj.leu.2400891

19. Greiner J, Götz M, Bunjes D, Hofmann S, Wais V. Immunological and clinical impact of manipulated and unmanipulated DLI after allogeneic stem cell transplantation of AML patients. J Clin Med. 2019;9(1):39. doi:10.3390/jcm9010039

20. Hofmann S, Götz M, Schneider V, et al. Donor lymphocyte infusion induces polyspecific CD8 + T-cell responses with concurrent molecular remission in acute myeloid leukemia with NPM1 mutation. J Clin Oncol. 2013;31(3):e44-e47. doi:10.1200/JCO.2011.41.1116

21. Maywald O, Buchheidt D, Bergmann J, et al. Spontaneous remission in adult acute myeloid leukemia in association with systemic bacterial infection - case report and review of the literature. Ann Hematol. 2004;83(3):189-194. doi:10.1007/s00277-003-0741-y

22. Adams S. Toll-like receptor agonists in cancer therapy. Immunotherapy. 2009;1(6):949-964. doi:10.2217/imt.09.70

\section{Publish your work in this journal}

The International Medical Case Reports Journal is an international, peer-reviewed open-access journal publishing original case reports from all medical specialties. Previously unpublished medical posters are also accepted relating to any area of clinical or preclinical science. Submissions should not normally exceed 2,000 words or 4 published pages including figures, diagrams and references. The manuscript management system is completely online and includes a very quick and fair peer-review system, which is all easy to use. Visit http://www.dovepress.com/testimonials.php to read real quotes from published authors. 